\title{
Towards a Connectomic Description of the Osteocyte Lacunocanalicular Network in Bone
}

\author{
Richard Weinkamer $^{1} \cdot$ Philip Kollmannsberger ${ }^{2} \cdot$ Peter Fratzl $^{1}$
}

Published online: 15 May 2019

(C) The Author(s) 2019

\begin{abstract}
Purpose of Review Osteocytes are the most abundant bone cells. They are completely encased in mineralized tissue, sitting inside lacunae that are connected by a multitude of canaliculi. In recent years, the osteocyte network has been shown to fulfill endocrine functions and to communicate with a number of other organs. This review addresses emerging knowledge on the connectome of the lacunocanalicular network in different types of bone tissue.

Recent Findings Recent advances in three-dimensional imaging technology started to reveal parameters that are well known from general theory to characterize the function of networks, such as network density, degree of nodes, or shortest path length through the network.

Summary The connectome of the lacunocanalicular network differs in some aspects between lamellar and woven bone and seems to change with age. More research is needed to relate network structure to function, such as intercellular transport or communication and its role in mechanosensation, as well as to understand the effect of diseases.
\end{abstract}

Keywords Connectome $\cdot$ Osteocyte network $\cdot$ Canaliculi $\cdot$ Image analysis $\cdot$ Image quantification $\cdot$ Fluid flow

\section{Introduction}

Osteocytes are the most abundant bone cells. They form a complex cell network within compact bone tissue being housed within a bone porosity consisting of canaliculi and lacunae, which form the lacunocanalicular network (LCN). While the general existence and micro-anatomy of the LCN has been known for a long time [1], it sparked renewed interest in the last years, due to the connections of the osteocyte network with other organs [ $2 \cdot$, 3•], and its importance for phosphate metabolism. In this context, osteocytes communicate with the kidney through the factor FGF23 [4], but also with the brain through the expression of leptin [5]. Interestingly, this communication between bone and other organs has already been postulated millenaries ago in the

This article is part of the Topical Collection on Osteocytes

Peter Fratzl

peter.fratzl@mpikg.mpg.de

1 Department of Biomaterials, Max Planck Institute of Colloids and Interfaces, 14424 Potsdam, Germany

2 Center for Computational and Theoretical Biology, Universität Würzburg, Campus Hubland Nord 32, 97074 Würzburg, Germany
Inner Canon of the Yellow Emperor (Huangdi Neijing), the classic text of Chinese medicine. According to this text, the human body is divided into five organ networks, one of them connecting the kidney with bone, marrow, and the brain [6]. Besides this interaction of osteocytes with other organs, the connectivity between osteocytes themselves is of crucial importance to understand their contribution to bone health. Osteocytes are regulating bone remodeling and, in particular, bone's mechanobiological adaptation, for example, through sensing fluid flow and the expression of sclerostin [7]. This means that the osteocyte network has to function as a (mechano)sensory organ which implies a complex communication between the cells [8]. While some known characteristics of the LCN, including the average number of canaliculi emerging from lacunae, have been recently reviewed $[9 \cdot 10]$, the overall network characteristics and, in particular, the connectome of the network, i.e., its "wiring diagram," received less attention. The aim of this short review is to summarize our emerging knowledge on the LCN connectome made possible by recent progress in imaging methodology. Since connectomics is already much further developed in neuroscience, the review mentions possible areas where bone researchers can learn from neuroscientists.

Conceptually, it is important to distinguish between the lacunocanalicular network (LCN) and the osteocyte network $(\mathrm{ON})$ as connected cell network (see Fig. 1 in [11•]). However, 
most of the functions of the osteocyte network can only be understood in the interplay between the "biological" cell network and the "material" porosity in the mineralized matrix [8]. In human osteons, canaliculi that are not oriented towards the Haversian canal were found to be co-aligned with the preferred matrix orientation [12]. The pericanalicular matrix in the immediate vicinity of the cell processes was shown to be disordered [13] and also more mineralized [14•] with an increased thickness of the mineral particles [15•] incorporated in the collagen matrix. This higher mineral content around canaliculi is remarkable in the context of the osteocytes' contribution to the calcium and phosphate metabolism. Recent evidence revived the almost forgotten idea of osteocytic osteolysis $[16,17]$. Due to the high surface area of the LCN [9•] and the small distance from the LCN to almost any point in the bone matrix $[18,19 \cdot]$, osteocytes have easy access to the bone mineral and are able to demineralize bone [20]. The role of osteocytes as mechanosensors and orchestrators of bone remodeling depends crucially on the interplay between cell network and porous network. The fluid flow hypothesis [21, 22] assumes that mechanical loading squeezes the interstitial bone fluid through the pericellular space between the cell processes and bodies and the canaliculi and lacunae. The osteocytes then sense the shear forces caused by the fluid flow, where cell processes seem to be more mechanosensitive than the cell body [23]. The details of the fluid flow and the resulting shear forces do not only depend on the connectivity and the irregular shape of the canaliculi, but also on how the cells deform due to the flow [24] and how the cell processes are anchored on the canaliculi walls [25].

\section{General Remarks on Connectomics and Network Function}

The highly interconnected and dense network of canaliculi has been compared to the network of neurons in the brain [26 $]$. For neurons, however, the function of the network (distributing signals among cells through synapses) is better known. In the field of neuroscience, describing the structure of the network in terms of neurons and their processes, as well as the synapses linking them, is seen as key for understanding higher-order functions such as sensory processing, motor output, or memory $[27 \bullet \bullet]$. Neural "connectomics" relates to the study of this structural and functional connectivity on multiple levels, including the connections between neurons and the interconnectedness of different brain regions. Connectomics studies in neuroscience have boosted the development of new high-end microscopy and image analysis methods in the last years [28] resulting in several recently published large-scale image datasets and cell-level connectomes of model organisms [29-31]. The obtained connectivity matrix (i.e., the description of which neurons are connected with each other, often without considering their spatial arrangement) has been successfully used to understand function in different model animals [32-35]. Additionally, a rich set of tools to quantify, visualize, and model neural networks has been developed [36]. Most of these methods are not specific to neurons and can, in principle, be adapted to other types of networks.

For the osteocyte network, the link between network structure and function is less obvious, and the spatial arrangement of the osteocyte network in the mineralized matrix cannot be neglected. Given the bulk of knowledge and methodologies available for the study of neural networks, it seems a promising research strategy to employ a "connectomics approach" to quantify differences between bones known to have a different response to loading conditions, for example, in diseased or aged organisms. Secondly, the connectomics data may be used to vet the multiple alleged functions of the osteocyte network by testing hypotheses regarding the efficiency of the network for the hypothesized functions.

\section{Recent Progress in Imaging the LCN Connectome}

Connectomics approaches are always intimately linked with experimental techniques to image the interconnectivity. In neuroscience, depending on the level of study, a distinction is made between microscopic mapping (i.e., neuron-to-neuron mapping using, for example, tract tracing), macroscopic mapping of major fiber bundles in the brain, and functional mapping using diverse magnetic resonance imaging (MRI) or optogenetics techniques [37]. The encasement of osteocytes in the mineralized bone matrix makes it more difficult to observe cell activities. But in terms of studying the LCN connectome, the encasement is partly an advantage, since the structure is cast in solid bone. The strong contrast in electron density between the mineralized matrix and the porosity of the LCN allows employing X-ray and electron imaging techniques [38•], for example. Another possibility is to invert the electron contrast by casting the LCN (e.g., by methacrylate) and then image the casting result by scanning electron microscopy after dissolution of the bone matrix [39].

To provide a data set of the LCN which can be analyzed in terms of its connectome, one needs to overcome the typical conflict between resolution and field of view. Indeed, three requirements have to be fulfilled: (i) the $\mathrm{LCN}$ has to be imaged in three dimensions to map accurately connections between canaliculi. Therefore, conventional light microscopy (LM), scanning electron microscopy (SEM), and atomic force microscopy (AFM) [40] are inadequate imaging methods for the task; (ii) the resolution of the imaging method has to be high enough so that canaliculi and their connections can be reliably traced. This excludes conventional absorption-based microcomputed tomography $(\mu \mathrm{CT})$, which remains, however, a 
powerful tool to study the shapes and spatial distributions of osteocyte lacunae [41]; (iii) the imaged bone volume has to be large enough to include a sufficient number of canaliculi with their interconnections. A single canaliculus with all its wall roughness has been imaged using TEM tomography [42], but this is clearly an insufficient field of view from a connectomics perspective. The resolution of X-ray tomography methods has been improved considerably [43, 44], using the intensive and partly coherent X-rays of synchrotrons. Xray phase nano-tomography $[14 \cdot, 45]$, and ptychographic $X$ ray computed tomography (PXCT) [46] were used to image the LCN with a voxel side length of 40-50 nm. An even higher resolution is possible with focused ion beam/scanning electron microscopy (FIB/SEM) [13, 47]. However, with all these high-resolution methods, the imaged bone volume typically includes a single or a few lacunae with their emerging canaliculi. Although these image data provide valuable insights in details of the LCN geometry and the surrounding matrix, it is obvious that they render a too small part of the LCN to be analyzed in terms of the connectome.

A powerful tool to image substantial parts of the canalicular network is a combination of staining and confocal laser scanning microscopy (CLSM) [15•]. The native (i.e., unembedded) bone sample is immersed in a solution containing a staining molecule like rhodamine. Confocal imaging gives image stacks with a distance of $0.3 \mu \mathrm{m}$, up to a depth of about $50 \mu \mathrm{m}$ due to the opaqueness of mineralized bone. With a typical CLSM setting, an image stack corresponds to an imaged volume of approximately $7 \times$ $10^{6} \mu^{3}$. While the diameter of canaliculi is too small to be resolved with light microscopy, their shape can be resolved in fluorescent microscopy because the distance separating them is typically larger than the light-microscopic resolution.

Important progress was also made in the imaging of not only the lacunocanalicular network, but also simultaneously of the osteocyte network by staining their cell membrane, nucleus, and cytoskeleton. In the procedure, mice were injected intravenously with lysine-fixable dextran and sacrificed a few minutes after the injection. The stain stays in place at the canalicular walls during the decalcification of the sample. After the embedding and immunostaining of the samples, multiplexed confocal imaging can be used to image various aspects of the osteocyte structure [48 ${ }^{\bullet}$.

A label-free method whose working principle is based on a sensitivity to interfaces is third-harmonic generation (THG) microscopy [49•]. Although the image quality is currently lower compared to CLSM, an advantage of THG is that already embedded samples and archeological artifacts that do not have a LCN which is accessible to stains can still be studied in terms of the canalicular network. THG has also been used for in vivo imaging of osteocytes in a mouse calvaria model $[50,51]$.
Image Analysis and Quantification of the LCN Connectome

An imaged bone volume with sufficiently many interconnected canaliculi has to run through an image analysis procedure before the LCN can be quantified in terms of its connectome. The first step in the image analysis is a binarization of the image into image voxels that belong to the LCN and the ones which do not by using either a globally or locally defined threshold value. The voxels representing the LCN are then segmented into voxels that belong to osteocyte lacunae and voxels that represent canaliculi. The segmentation criterion is naturally based on the very different "bulkiness" of lacunae and canaliculi. For the analysis of the lacunae, different frameworks have been developed which are based on a fitting of the lacuna by an ellipsoid $[52,53]$. The resulting ellipsoids can then be quantified in terms of their shape (prolateness, oblateness), their orientation in relation to a pre-defined coordinate system, and their spatial relation to neighboring lacunae.

More intricate is the analysis of the canaliculi since the current imaging technology demands a choice to be made. Either the imaging resolution is high enough that the canaliculi are rendered reliably, but then the imaged volume is too small for a connectome approach. In this case, the quantification of the LCN structure can be continued in analogy to the analysis of the vascular network [54], which follows the standard analysis of the trabecular bone structure. Quantities that can be evaluated are canalicular volume fraction (Cn.V/TV) with TV the total bone volume, canalicular thickness (Cn.Th), canalicular spacing (Cn.Sp), and canalicular structure model index (Cn.SMI) [47]. Other local parameters include the average number of canaliculi emerging from a lacuna.

The alternative choice is methods which combine staining and confocal laser scanning microscopy (CLSM) (Fig. 1). In CLSM, the signal of the stain is blurred also due to penetration of the stain into the bone matrix. Attempts to quantify the canalicular volume therefore suffer from partial volume effect and easily overestimate this volume [55, 56]. However, CLSM provides reliable information on the location of the canaliculi and their interconnectivity, which can be used to infer the topological structure or "connectome" of the canalicular network (Fig. 1), although there is no information on actual cell-cell connections. To obtain the structure or "skeleton" of the LCN, the location of the canaliculi and the junctions ("nodes") between them has to be extracted. A thinning algorithm is applied to the binarized and segmented image data to obtain the center line or medial axis of the canaliculi. In case of low signal-to-noise ratio, adaptive or topological thresholding (Kerschnitzki 2013) or machine learning with pixel classifiers or convolutional neural networks $(\mathrm{CNN})$ [57] can be applied to prevent artifacts such as false negative (missing) or false positive (non-existing) links between canaliculi. Alternatively, the initial thresholding step can even be 
Fig. 1 Below: work flow from an image stack obtained by confocal laser scanning microscopy (CLSM) (gray, left) to a binarized image of the LCN (red, middle) to a mathematical network consisting of edges (i.e., canaliculi) and nodes (i.e., lacunae and meeting points of canaliculi) (blue, right). The image at the top shows a magnification of the volume encircled by the white box
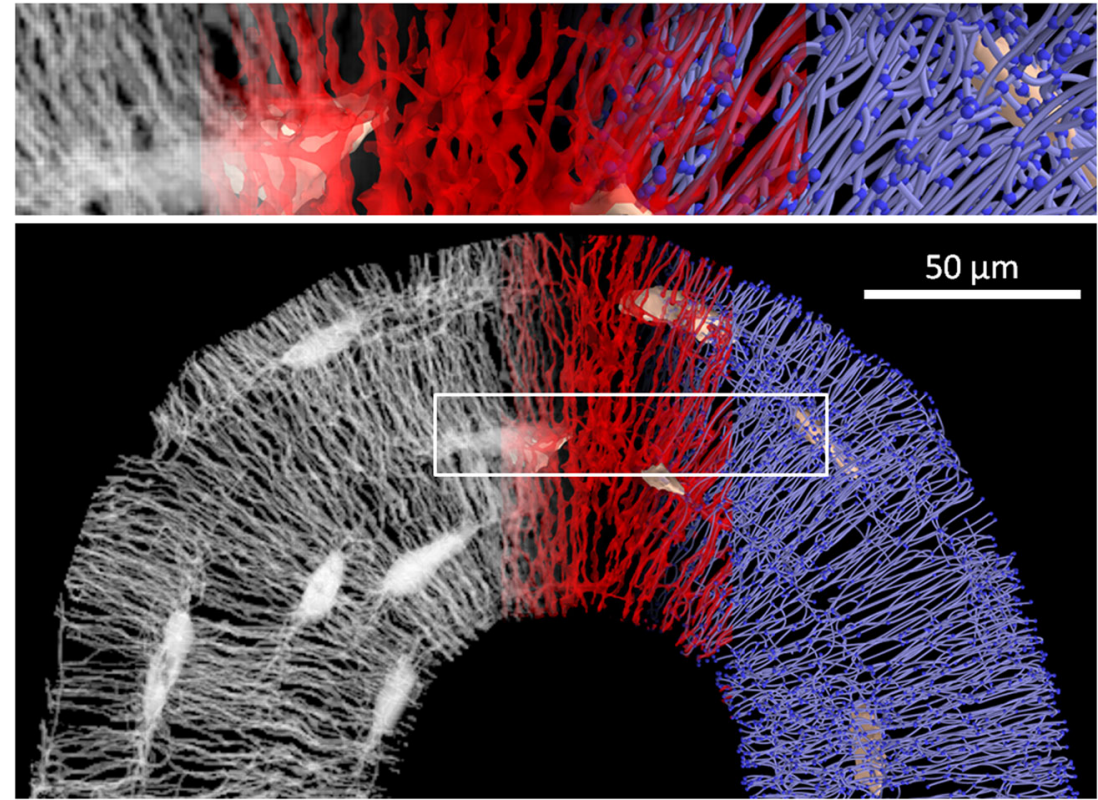

omitted by tracing canaliculi in the raw images to obtain the skeleton of the network [58]. Finally, the skeleton is converted into a mathematical network ("graph") consisting of nodes (where at least three canaliculi meet and, therefore, including lacunae) and edges (canaliculi linking two nodes) (Fig. 1) using specialized software $[18,19 \bullet, 59]$.

The LCN as a network consisting of nodes and edges can be described on different levels of realistic rendering. In the most abstract form, the network is represented by a "connectivity matrix" $\boldsymbol{A}$, where each row and column corresponds to one node and the entries $a_{\mathrm{ij}}$ correspond either to the number of edges or the length of the single edge between the two nodes $i$ and $j$. Since connections via canaliculi in the LCN are virtually restricted to nodes in the neighborhood of a specific node, most entries in the connectivity matrix are zero, i.e., the matrix is sparse. Alternatively, the LCN is viewed as a spatial network described by the actual position of the individual nodes and edges as curved lines in space. This representation of the LCN as a spatial network allows to tackle, for example, questions about the orientation of canaliculi towards larger structures in bones like the endosteal or periosteal surfaces or the Haversian canal in osteonal bone [12]. The close interrelation between the LCN and the surrounding bone matrix raises the question whether the network definition should be further extended for the LCN by taking parameters into account which characterize the surrounding matrix (e.g., preferred collagen matrix orientation, mineral content).

After the network structure of nodes and edges has been extracted from the images, various properties can be calculated to quantify and compare different networks (see Table 1). Since the LCN is a physical network in space, one can extract geometric measures (such as density or distances) as well as topological measures (such as node degree or clustering coefficients). The latter can be derived from the connectivity matrix [60]. The "betweenness" of a node is the number of shortest paths of the network running through that particular node. For the osteocyte LCN, nodes with high betweenness line up between cell lacunae, indicating the existence of "important" paths through the network (Fig. 2 and $[61 \bullet \bullet])$. The parameter of small worldness is defined as the ratio of the clustering coefficient to the average shortest path length relative to a random network and characterizes how efficient a network is in connecting distant nodes with as few edges as possible. All these parameters are well established in the field of "network science," and a large body of existing literature allows to compare results for different types of networks, from neural networks to the internet [60, $62]$. Two caveats have to be added here: firstly, generic network properties often do not account for the spatial embedding of the network [63]. For example, while in a generic network all nodes are "equivalent," in a spatial network, long-range connections between nodes are rather unlikely. Secondly, many concepts in network research are based on simple generative models of random or regular networks. These rule-based models usually lack any biological plausibility and should be, therefore, taken with care.

\section{LCN Connectome in Different Bone Tissues}

Published results that characterize the connectome of the canalicular or the osteocyte network are rather scarce. An important reference value for the denseness of the LCN in healthy humans was established recently on a study of femoral osteonal bone. In osteons, the average canalicular density was found to be Ca.Dn $=0.074 \pm 0.015 \mu \mathrm{m} / \mu \mathrm{m}^{3}$, which 
Table 1 Selection of parameters defining the connectome of the lacunocanalicular network

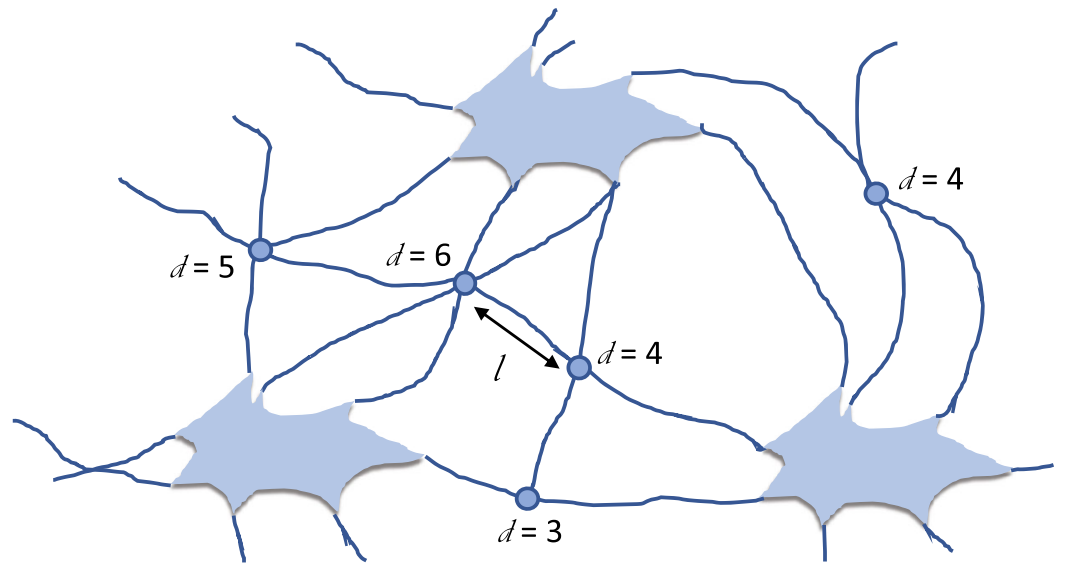

\begin{tabular}{|c|c|c|}
\hline Parameter & & Unit \\
\hline Node degree & Number of edges per node ( $d$ in the sketch) & - \\
\hline Edge length & $\begin{array}{l}\text { Distance between two nodes ( } 1 \text { in the sketch) considering the tortuosity of } \\
\text { the canaliculi }\end{array}$ & $\mu \mathrm{m}$ \\
\hline Node density & Number of nodes per unit volume (cell lacunae being considered as nodes) & $\mu \mathrm{m}^{-3}$ \\
\hline Canalicular density & Total length of canaliculi per unit volume & $\mu \mathrm{m}^{-2}$ \\
\hline Lacunar density & Number of osteocyte lacunae per unit volume & $\mu \mathrm{m}^{-3}$ \\
\hline Distance to bone matrix & Average closest distance from any point in the bone tissue to the network & $\mu \mathrm{m}$ \\
\hline Degree of edge alignment & $\begin{array}{l}\text { The alignment can be defined either with respect to a fixed coordinate } \\
\text { system } \\
\text { (e.g., the Haversian canal) or as mutual alignment of the canaliculi }\end{array}$ & - \\
\hline Clustering coefficient & $\begin{array}{l}=0 \text { if none of the neighbors of a node are linked by canaliculi, and }=1 \text { if } \\
\text { all possible links between neighbors of a node exist }\end{array}$ & - \\
\hline Average shortest path & $\begin{array}{l}\text { Global network property that denotes how many nodes have to be traversed } \\
\text { on average to reach any node in the network from any other node }\end{array}$ & - \\
\hline Betweenness of a node & $\begin{array}{l}\text { Number of shortest paths of the network running through that particular } \\
\text { node }\end{array}$ & - \\
\hline Small worldness & $\begin{array}{l}\text { Ratio of the clustering coefficient to the average shortest path relative to a } \\
\text { random network }\end{array}$ & - \\
\hline
\end{tabular}

means that a cubic centimeter of osteonal bone contains on average canaliculi of a total length of $74 \mathrm{~km}$. Assuming the canaliculi of a cylindrical shape with an average diameter of Ca.Dm $=315 \mathrm{~nm}[9,45]$, this corresponds to a canalicular porosity of $\frac{\mathrm{Ca} . \mathrm{V}}{\mathrm{TV}}=\frac{D^{2}}{4} \cdot \pi \cdot \mathrm{Ca} . \mathrm{Dn}=0.0058$, i.e., the canalicular porosity is only slightly larger than $0.5 \%$. For $80 \%$ of the bone, the distance to the closest canaliculus was found to be smaller than $2.8 \mu \mathrm{m}$. In other species, these impressive numbers were even surpassed. The highest values of the canalicular density up to now were found in the fibrolamellar bone of sheep (femur, mid-diaphysis), Ca.Dn $=0.19 \pm 0.01 \mu \mathrm{m} / \mu^{3}$. In between the human and the ovine network density, the density in the particularly disordered part of murine cortical bone was determined to be Ca.Dn $=0.10 \pm 0.01 \mu \mathrm{m} / \mu \mathrm{m}^{3}$. The denser network in sheep entailed that about $95 \%$ of the bone matrix was found to be within $2 \mu \mathrm{m}$ from the network. In the same study, a network analysis showed that this dense, regularly organized network in sheep is less connected, but more efficiently organized compared to the network in irregular and fast-growing bone tissue from mice. The statistical topological properties such as edge density (fraction of possible connections between nodes that actually exist) as well as edge length and node degree distribution are identical in both network types, indicating that despite pronounced differences at the tissue level, the topological architecture of the osteocyte canalicular network at the subcellular level may be independent of species and bone type $[61 \bullet \bullet]$.

\section{Influence of Disease on the LCN Connectome}

Already more than a decade ago, histological observations pointed to changes in the LCN's architecture for the most prominent bone diseases [64]. Beside the orientation and the tortuosity of the canaliculi, the connectivity of the network was altered. In particular, a reduced connectivity was observed 


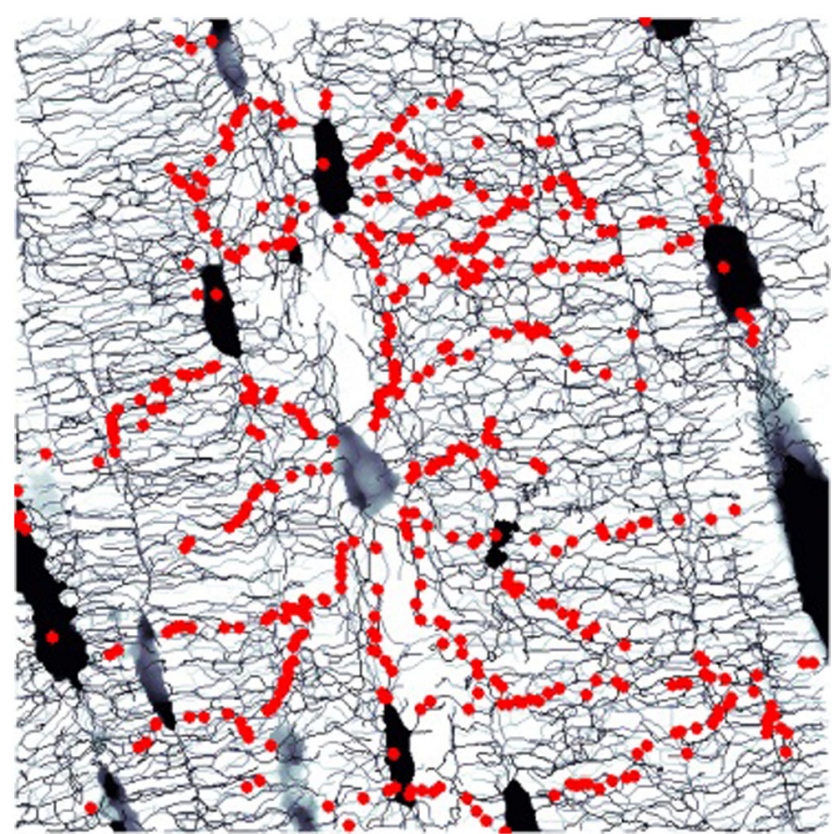

Fig. 2 Example of a parameter of network theory applied to the lacunocanalicular network (LCN): red dots show nodes in the network with a high value of betweenness (see Table 1). These nodes line up to form "highways" through the LCN [61]

in osteoporotic and osteoarthritic bone, while in case of osteomalacia, the LCN connectivity was not reduced [64, 65]. Since these studies were not quantitative, the statistical significance of these findings still has to be tested. Using the rat ovariectomy (OVX) model of postmenopausal osteoporosis, the rats did not display a reduced number of canaliculi emerging from the lacunae compared to the control animals, although the diameter of the canaliculi was found to be increased [55]. Using a Wistar rat model of glucocorticoid-induced osteoporosis (GIO), the difference in network architecture to control animals was rather subtle, with no statistically significant difference for the total number of canaliculi emerging from the lacuna, but with a significant difference in the number of canaliculi per surface area of the lacuna [46]. The perlecan-deficient Hypo mouse had a reduced canalicular number density, while the Akita mouse, a model for spontaneous type 1 diabetes, did not show any statistically significant changes in the network architecture [66]. Imaging the osteocyte network (ON) of osteocytes, the architecture of the network in high fat-fed diabetic mice was found to have a higher mean node degree, while the average edge length was decreased compared to lean control animals [67].

\section{Aging and Formation of the LCN Connectome}

An important question is whether changes in the LCN architecture and connectome with age [68] contribute to age-related bone loss and to a reduction in bone's mechanosensitivity as observed in mice [69]. A recent study on a standard C57BL/6 mouse model with mice aged between 5 and 22 months investigated the influence of age on both the osteocyte network and the lacunocanalicular network using multiplexed confocal imaging $[70 \bullet \bullet]$. Focusing on local connectivity of the network structures, a strong reduction in the number of cell processes, in particular in female animals, was observed with age. Also, the number of canaliculi per lacuna decreased significantly with age. While in young animals, the number of canaliculi outnumbers the number of cell processes only by a factor of $1.2-1.4$, this factor increases with age to $1.5-1.7$; therefore, relatively more canaliculi remain unoccupied by cell processes in older animals [70••].

Studies on human bone also indicate a reduction in the network density [56]. As human bone is an example of bone undergoing substantial remodeling during a lifetime, the distinction between the age of the individual and tissue age is crucial. The report of large volumes in human osteons without accessible LCN [19॰] provides the view that an architectural degeneration of the LCN with age is a spatially heterogeneous process rather than a homogeneous thinning of the network. The observation of cell processes without cell body shows the possibility that cell remnants can still be present after cell death or apoptosis $\left[70^{\bullet}\right]$. On the long run, the absence of a living osteocyte will most likely result in micropetrosis [71] and a local clogging of the LCN.

More research is also needed to understand how the $\mathrm{LCN}$ is formed in the first place. In comparison between the osteocyte network of embryonic mice with mice 6 weeks of age, the embryonic mice had fewer cell processes radiating from an osteocyte than the older animals [72]. Factors that might influence network formation can be both cell-intrinsic properties as well as the tissue environment. For example, cell age and differentiation might change the dynamics of cell processes, or bone material properties could regulate their outgrowth velocity. Also, mechanical stimulation has to be considered as a potential controlling factor in the formation process of the network, where mechanical forces could not only result from muscle action, but also from changes in the osmotic pressure resulting in water-generated stresses in the collagen matrix [73]. To really understand the formation process of the LCN formation, the aim has to be to shift from the static network images available now, to dynamic movies of the formation process. Here, computer simulations can be a helpful tool $[74,75]$. Based on assumptions about the local growth process, such as how often osteocyte processes branch or how they respond to tissue orientation, soluble gradients, mechanical stimulation, or physical constraints, a growing network can be simulated. The resulting network can be quantified by deriving the same properties as from images of the LCN. Subsequently, the growth rules in the model can be varied and the process iterated until the simulation results in a plausible network pattern. To confirm the findings by such computer 
simulations, growing networks should be imaged and quantified ideally in a time-resolved manner, for example, using live cell microscopy on short timescales, or time lapse in vivo microscopy for longer timescales.

\section{Conclusions}

Connectomics of the osteocytic lacunocanalicular network is only starting to emerge. More research in this direction is needed to help clarifying the function of the osteocyte network. In light of the putative multifunctionality of the osteocyte network, this is a long-term perspective. As the first steps in this direction, fluid flow analyses through the LCN have been performed to understand the influence of network architecture on fluid flow-mediated mechanosensation. However, up to now, the used LCN connectomes were still highly idealized in these approaches [76-78].

A more accessible goal would be to resolve the question of whether the LCN connectome can be used as a fingerprint of different types of bone tissue. How much can we learn from a LCN connectome in terms of skeletal site, bone type, sex, and age, and does a closer look at the LCN provide new possibilities to diagnose bone diseases? If the LCN can be imaged in historical bone samples, this aspect could also be of major interest for anthropologists. On the basis of a sound characterization of the LCN connectome in different bone types, a next step could be to try to positively influence the network architecture, e.g., by applying mechanical stimulation or drug treatment.

Work on the LCN connectome can clearly benefit from other fields, such as neural or vascular networks, and the results characterizing the different connectomes could be compared to get deeper insights in how network-like structures form and are adapted for communication and transport. In particular, the comparison between the osteocyte network and the neural network seems promising, not only because of a certain similarity in the appearance of the two network structures. Neural networks possess a directionality with signals being transmitted from the presynaptic to the postsynaptic neuron and the network clearly performs a "computational" function. In bone with the presence of the two networks, the ON and LCN, with one inside the other, and the remaining space filled with interstitial fluid, the network structure seems to be even more favorable for function. In particular, osteocytes have been reported to use the LCN dynamically by expanding and contracting their cell bodies in the lacunae and moving their cell processes in and out of the canaliculi $[38,79]$. With some regulation, this movement of cell processes could be used to influence the direction of the fluid flow, or even act as a system with valves, if the cell process could completely block the fluid flow within a canaliculus. If osteocytes would actively manipulate the fluid flow through the LCN in such a way, this would allow communication independent of direct cell- cell contact via gap junctions, mediated by fluid flow. This raises the interesting question whether osteocyte networks may even be solving computational tasks [22, 26], e.g., related to mechanical adaptation. Even without such speculations, the next decade of research on the connectome of the osteocyte and the lacunocanalicular network will not only help clarifying the function of the most abundant bone cell, but may likely also provide us with some surprises.

Acknowledgments The authors want to thank Alexander van Tol for his help with Fig. 1. RW and PF are members of the Excellence Cluster "Matters of Activity - Image Space Material" at Humboldt University Berlin supported by the German Research Foundation (DFG).

Funding Information Open access funding provided by Max Planck Society.

\section{Compliance with Ethical Standards}

Conflict of Interest The authors declare that they have no conflict of interest.

Human and Animal Rights and Informed Consent This article does not contain any studies with human or animal subjects performed by any of the authors.

Open Access This article is distributed under the terms of the Creative Commons Attribution 4.0 International License (http:// creativecommons.org/licenses/by/4.0/), which permits unrestricted use, distribution, and reproduction in any medium, provided you give appropriate credit to the original author(s) and the source, provide a link to the Creative Commons license, and indicate if changes were made.

\section{References}

Papers of particular interest, published recently, have been highlighted as:

- Of importance

•• Of major importance

1. Tomes J, De Morgan CG. IV. Observations on the structure and development of bone. Philos Trans R Soc Lond. 1853;143:109-39.

2. Bonewald LF. The amazing osteocyte. J Bone Miner Res. 2011;26(2):229-38 Still an excellent starting point to learn about the biology and function of osteocytes.

3. Schaffler MB, Cheung WY, Majeska R, Kennedy O. Osteocytes: Master orchestrators of bone. Calcif Tissue Int. 2014;94(1):5-24 Provides an excellent overview over osteocytes' role in mechanotransduction.

4. Bonewald LF. Osteocytes as dynamic multifunctional cells. Ann N Y Acad Sci. 2007;1116:281-90.

5. Hamrick MW. Leptin, bone mass, and the thrifty phenotype. J Bone Miner Res. 2004;19(10):1607-11.

6. Fruehauf HO. The five organ networks of Chinese medicine. Portland: National College of Naturopathic Medicine; 1997.

7. Moester M, Papapoulos S, Löwik C, Van Bezooijen R. Sclerostin: current knowledge and future perspectives. Calcif Tissue Int. 2010;87(2):99-107. 
8. Jacobs CR, Temiyasathit S, Castillo AB. Osteocyte mechanobiology and pericellular mechanics. Annu Rev Biomed Eng. 2010;12:369-400.

9. Buenzli PR, Sims NA. Quantifying the osteocyte network in the human skeleton. Bone. 2015;75:144-50 Review that provides a lot of useful values characterizing the lacunocanalicular network.

10. Cardoso L, Fritton SP, Gailani G, Benalla M, Cowin SC. Advances in assessment of bone porosity, permeability and interstitial fluid flow. J Biomech. 2013;46(2):253-65.

11. Schneider P, Meier M, Wepf R, Muller R. Towards quantitative 3D imaging of the osteocyte lacuno-canalicular network. Bone. 2010;47(5):848-58 Introduces with FIB/SEM an important new 3D imaging technique to study the $L C N$.

12. Repp F, Kollmannsberger P, Roschger A, Berzlanovich A, Gruber GM, Roschger P, et al. Coalignment of osteocyte canaliculi and collagen fibers in human osteonal bone. J Struct Biol. 2017;199(3):177-86.

13. Reznikov N, Shahar R, Weiner S. Three-dimensional structure of human lamellar bone: the presence of two different materials and new insights into the hierarchical organization. Bone. 2014;59:93104.

14. Hesse B, Varga P, Langer M, Pacureanu A, Schrof S, Mannicke N, et al. Canalicular network morphology is the major determinant of the spatial distribution of mass density in human bone tissue: evidence by means of synchrotron radiation phase-contrast nano-CT. J Bone Miner Res. 2015;30(2):346-56 State-of-the-art synchrotron tomography shows increased mineral content around canaliculi

15. Kerschnitzki M, Wagermaier W, Roschger P, Seto J, Shahar R, Duda GN, et al. The organization of the osteocyte network mirrors the extracellular matrix orientation in bone. J Struct Biol. 2011;173(2):303-11 The study shows the interaction between LCN architecture and the surrounding bone matrix.

16. Belanger LF. Osteocytic osteolysis. Calcif Tissue Res. 1969;4(1):1$\&$.

17. Tsourdi E, Jähn K, Rauner M, Busse B, Bonewald LF. Physiological and pathological osteocytic osteolysis. J Musculoskelet Neuronal Interact. 2018;18(3):292-303.

18. Kerschnitzki M, Kollmannsberger P, Burghammer M, Duda GN, Weinkamer R, Wagermaier W, et al. Architecture of the osteocyte network correlates with bone material quality. J Bone Miner Res. 2013;28(8):1837-45.

19. Repp F, Kollmannsberger P, Roschger A, Kerschnitzki M, Berzlanovich A, Gruber G, et al. Spatial heterogeneity in the canalicular density of the osteocyte network in human osteons. Bone Rep. 2017;6:101-8 Provides reference values for the LCN properties in healthy osteonal bone.

20. Nango N, Kubota S, Hasegawa T, Yashiro W, Momose A, Matsuo K. Osteocyte-directed bone demineralization along canaliculi. Bone. 2016;84:279-88.

21. Burger EH, Klein-Nulend J. Mechanotransduction in bone - role of the lacuno-canalicular network. FASEB J. 1999;13(9001):S101S12.

22. Cowin SC, Moss ML. Mechanosensory mechanisms in bone. In: Cowin SC, editor. Bone mechanics handbook. 2nd ed. Boca Raton: CRC Press; 2001.

23. Thi MM, Suadicani SO, Schaffler MB, Weinbaum S, Spray DC. Mechanosensory responses of osteocytes to physiological forces occur along processes and not cell body and require $\alpha \mathrm{V} \beta 3$ integrin. Proc Natl Acad Sci. 2013;110(52):21012-7.

24. Verbruggen SW, Vaughan TJ, McNamara LM. Fluid flow in the osteocyte mechanical environment: a fluid-structure interaction approach. Biomech Model Mechanobiol. 2014;13(1):85-97.
25. Wang YL, McNamara LM, Schaffler MB, Weinbaum S. A model for the role of integrins in flow induced mechanotransduction in osteocytes. Proc Natl Acad Sci U S A. 2007;104(40):15941-6.

26. Turner C, Robling A, Duncan R, Burr D. Do bone cells behave like a neuronal network? Calcif Tissue Int. 2002;70(6):435-42 Early but still interesting work that discusses experimental evidence for memory-like mechanisms in the osteocyte network.

27.• Meinertzhagen IA. Of what use is connectomics? A personal perspective on the Drosophila connectome. J Exp Biol. 2018;221(10): jeb164954 Nice overview article on the history, current state, and future perspective of connectomics in the Drosophila brain from one of the pioneers in the field.

28. Helmstaedter MJ. Connectomics at cellular precision. eNeuroforum. 2016;22(3):45-7.

29. Oh SW, Harris JA, Ng L, Winslow B, Cain N, Mihalas S, et al. A mesoscale connectome of the mouse brain. Nature. 2014;508(7495):207.

30. Hildebrand DGC, Cicconet M, Torres RM, Choi W, Quan TM, Moon J, et al. Whole-brain serial-section electron microscopy in larval zebrafish. Nature. 2017;545(7654):345.

31. Eichler K, Li F, Litwin-Kumar A, Park Y, Andrade I, SchneiderMizell CM, et al. The complete connectome of a learning and memory centre in an insect brain. Nature. 2017;548(7666):175 The first complete wiring diagram of a higher-order circuit at synaptic resolution, the Drosophila larval mushroom body, obtained by a large collaboration over many years.

32. Yan G, Vértes PE, Towlson EK, Chew YL, Walker DS, Schafer WR, et al. Network control principles predict neuron function in the Caenorhabditis elegans connectome. Nature. 2017;550(7677):519 23 Example of how connectomics can be applied to predict neuronal function for locomotion in $C$. elegans worms using control theory.

33. Bezares-Calderon LA, Berger J, Jasek S, Veraszto C, Mendes S, Guehmann M, et al. Neural circuitry of a polycystin-mediated hydrodynamic startle response for predator avoidance. eLife. 2018;7: e36262.

34. Svara FN, Kornfeld J, Denk W, Bollmann JH. Volume EM reconstruction of spinal cord reveals wiring specificity in speed-related motor circuits. Cell Rep. 2018;23(10):2942-54.

35. Wanner AA, Genoud C, Masudi T, Siksou L, Friedrich RW. Dense EM-based reconstruction of the interglomerular projectome in the zebrafish olfactory bulb. Nat Neurosci. 2016;19(6):816-25.

36. Scheffer LK. Analysis tools for large connectomes. Front Neural Circuits. 2018;12.

37. Fenno L, Yizhar O, Deisseroth $\mathrm{K}$. The development and application of optogenetics. Annu Rev Neurosci. 2011;34:389-412.

38. Webster DJ, Schneider P, Dallas SL, Müller R. Studying osteocytes within their environment. Bone. 2013;54(2):285-95 Clear review about imaging techniques of the osteocyte and lacunocanalicular network.

39. Okada S, Yoshida S, Ashrafi SH, Schraufnagel DE. The canalicular structure of compact bone in the rat at different ages. Microsc Microanal. 2002;8(2):104-15.

40. Lin Y, Xu S. AFM analysis of the lacunar-canalicular network in demineralized compact bone. J Microsc. 2011;241(3):291-302.

41. Dong P, Haupert S, Hesse B, Langer M, Gouttenoire PJ, Bousson V, et al. 3D osteocyte lacunar morphometric properties and distributions in human femoral cortical bone using synchrotron radiation micro-CT images. Bone. 2014;60:172-85.

42. Kamioka H, Murshid SA, Ishihara Y, Kajimura N, Hasegawa T, Ando R, et al. A method for observing silver-stained osteocytes in situ in 3- $\mu \mathrm{m}$ sections using ultra-high voltage electron microscopy tomography. Microsc Microanal. 2009;15(5):377-83.

43. Langer M, Peyrin F. 3D X-ray ultra-microscopy of bone tissue. Osteoporos Int. 2016;27(2):441-55. 
44. Goggin P, Zygalakis K, Oreffo R, Schneider P. High-resolution 3D imaging of osteocytes and computational modelling in mechanobiology: insights on bone development, ageing, health and disease. Eur Cell Mater. 2016;31:264-95.

45. Varga P, Hesse B, Langer M, Schrof S, Mannicke N, Suhonen H, et al. Synchrotron X-ray phase nano-tomography-based analysis of the lacunar-canalicular network morphology and its relation to the strains experienced by osteocytes in situ as predicted by casespecific finite element analysis. Biomech Model Mechanobiol. 2015;14(2):267-82.

46. Ciani A, Toumi H, Pallu S, Tsai EH, Diaz A, Guizar-Sicairos M, et al. Ptychographic X-ray CT characterization of the osteocyte lacuno-canalicular network in a male rat's glucocorticoid induced osteoporosis model. Bone Rep. 2018;9:122-31.

47. Schneider P, Meier M, Wepf R, Muller R. Serial FIB/SEM imaging for quantitative $3 \mathrm{D}$ assessment of the osteocyte lacuno-canalicular network. Bone. 2011;49(2):304-11.

48. Kamel-ElSayed SA, Tiede-Lewis LM, Lu Y, Veno PA, Dallas SL. Novel approaches for two and three dimensional multiplexed imaging of osteocytes. Bone. 2015;76:129-40 Important technical paper that shows possibilities to study the interplay between the LCN and $O N$ by imaging both in the same bone sample.

49. Genthial R, Beaurepaire E, Schanne-Klein M-C, Peyrin F, Farlay D, Olivier C, et al. Label-free imaging of bone multiscale porosity and interfaces using third-harmonic generation microscopy. Sci Rep. 2017;7(1):3419 Demonstrates how nonlinear optics can be used to image the $\mathrm{LCN}$ without labeling.

50. Tokarz D, Cisek R, Wein MN, Turcotte R, Haase C, S-CA Y, et al. Intravital imaging of osteocytes in mouse calvaria using third harmonic generation microscopy. PLoS One. 2017;12(10):e0186846.

51. Wu P-C, Shen Y-F, Sun C-K, Lin CP, Liu T-M. Harmonic generation microscopy of bone microenvironment in vivo. Opt Commun. 2018;422:52-5.

52. Heveran CM, Rauff A, King KB, Carpenter RD, Ferguson VL. A new open-source tool for measuring 3D osteocyte lacunar geometries from confocal laser scanning microscopy reveals age-related changes to lacunar size and shape in cortical mouse bone. Bone. 2018;110:115-27.

53. Mader KS, Schneider P, Muller R, Stampanoni M. A quantitative framework for the 3D characterization of the osteocyte lacunar system. Bone. 2013;57(1):142-54.

54. Cooper DM, Turinsky AL, Sensen CW, Hallgrímsson B. Quantitative 3D analysis of the canal network in cortical bone by micro-computed tomography. Anat Rec B New Anat. 2003;274(1): 169-79.

55. Sharma D, Ciani C, Marin PAR, Levy JD, Doty SB, Fritton SP. Alterations in the osteocyte lacunar-canalicular microenvironment due to estrogen deficiency. Bone. 2012;51(3):488-97.

56. Ashique A, Hart L, Thomas C, Clement J, Pivonka P, Carter Y, et al. Lacunar-canalicular network in femoral cortical bone is reduced in aged women and is predominantly due to a loss of canalicular porosity. Bone Rep. 2017;7:9-16.

57. Litjens G, Kooi T, Bejnordi BE, Setio AAA, Ciompi F, Ghafoorian $\mathrm{M}$, et al. A survey on deep learning in medical image analysis. Med Image Anal. 2017;42:60-88.

58. Acciai L, Soda P, Iannello G. Automated neuron tracing methods: an updated account. Neuroinformatics. 2016;14(4):353-67.

59. Nunez-Iglesias J, Blanch AJ, Looker O, Dixon MW, Tilley L. A new Python library to analyse skeleton images confirms malaria parasite remodelling of the red blood cell membrane skeleton. PeerJ. 2018;6:e4312.

60. Kaiser M. A tutorial in connectome analysis: topological and spatial features of brain networks. Neuroimage. 2011;57(3):892-907.
61.• Kollmannsberger P, Kerschnitzki M, Repp F, Wagermaier W, Weinkamer R, Fratzl P. The small world of osteocytes: connectomics of the lacuno-canalicular network in bone. New J Phys. 2017;19:073019 Proves the potential of a connectomic approach by analyzing ovine lamellar bone and murine woven bone.

62. Clauset A, Tucker E, Sainz M. The Colorado index of complex networks. https://iconcolorado.edu/. 2016.

63. Barthélemy MJPR. Spatial networks. 2011;499(1-3):1-101.

64. Tate MK, Tami A, Bauer T, Knothe U. Micropathoanatomy of osteoporosis: indications for a cellular basis of bone disease. Adv Osteopor Fract Manage. 2002;2(1):9-14.

65. Tate MLK, Adamson JR, Tami AE, Bauer TW. The osteocyte. Int J Biochem Cell Biol. 2004;36(1):1-8.

66. Lai X, Price C, Modla S, Thompson WR, Caplan J, Kirn-Safran $\mathrm{CB}$, et al. The dependences of osteocyte network on bone compartment, age, and disease. Bone Res. 2015;3:15009.

67. Mabilleau G, Perrot R, Flatt PR, Irwin N, Chappard D. High fat-fed diabetic mice present with profound alterations of the osteocyte network. Bone. 2016;90:99-106.

68. Hemmatian H, Bakker AD, Klein-Nulend J, van Lenthe GH. Aging, osteocytes, and mechanotransduction. Current Osteoporos Rep. 2017;15(5):401-11.

69. Razi H, Birkhold AI, Weinkamer R, Duda GN, Willie BM, Checa S. Aging leads to a dysregulation in mechanically driven bone formation and resorption. J Bone Miner Res. 2015;30(10):1864-73.

70.• Tiede-Lewis LM, Xie Y, Hulbert MA, Campos R, Dallas MR, Dusevich V, et al. Degeneration of the osteocyte network in the C57BL/6 mouse model of aging. Aging (Albany NY). 2017;9(10):2190-208 An impressive study on the effect of age on both the LCN and $\mathrm{ON}$ in mice.

71. Frost HM. Micropetrosis. J Bone Joint Surg Am. 1960;42(1):144 50.

72. Sugawara Y, Kamioka H, Ishihara Y, Fujisawa N, Kawanabe N, Yamashiro T. The early mouse 3D osteocyte network in the presence and absence of mechanical loading. Bone. 2013;52(1):18996.

73. Masic A, Bertinetti L, Schuetz R, Chang S-W, Metzger TH, Buehler MJ, et al. Osmotic pressure induced tensile forces in tendon collagen. Nat Commun. 2015;6:5942.

74. Kaiser M. Mechanisms of connectome development. Trends Cogn Sci. 2017;21(9):703-17.

75. Taylor-King JP, Basanta D, Chapman SJ, Porter MA. Mean-field approach to evolving spatial networks, with an application to osteocyte network formation. Phys Rev E. 2017;96(1):012301.

76. Gururaja S, Kim H, Swan C, Brand R, Lakes R. Modeling deformation-induced fluid flow in cortical bone's canalicularlacunar system. Ann Biomed Eng. 2005;33(1):7-25.

77. Anderson EJ, Kreuzer SM, Small O, Tate MLK. Pairing computational and scaled physical models to determine permeability as a measure of cellular communication in micro-and nanoscale pericellular spaces. Microfluid Nanofluid. 2008;4(3): 193-204.

78. Mishra S, Tate MLK. Effect of lacunocanalicular architecture on hydraulic conductance in bone tissue: implications for bone health and evolution. Anat Rec A Discov Mol Cell Evol Biol. 2003;273a(2):752-62.

79. Veno P, Nicolella D, Sivakumar P, Kalajzic I, Rowe D, Bonewald L et al., editors. Live imaging of osteocytes within their lacunae reveals cell body and dendrite motions. J Bone Miner Res; 2006.

Publisher's Note Springer Nature remains neutral with regard to jurisdictional claims in published maps and institutional affiliations. 